\title{
THz-conductivity of CVD graphene on different substrates
}

\author{
Daniel Gabriel ${ }^{1}$, Bernat Sempere ${ }^{2}$, Carles Colominas ${ }^{2}$, Núria Ferrer-Anglada ${ }^{*}, 1$ \\ ${ }^{1}$ Applied Physics Department, Universitat Politècnica de Catalunya (UPC), Campus Nord B4, J. Girona 3-5, 08034 Barcelona, Spain \\ ${ }^{2}$ Materials Engineering Group, IQS School of Engineering, Universitat Ramon Llull, Via Augusta 390, 08017 Barcelona, Spain
}

Received ZZZ, revised ZZZ, accepted ZZZ

Published online ZZZ (Dates will be provided by the publisher.)

Keywords graphene, IR-UV-Vis spectroscopy, high frequency conductivity, THz-time domain spectroscopy, transmittance.

* Corresponding author: e-mail nuria.ferrer@upc.edu, Phone: +34 934016 880, Fax: +34 934016090

\begin{abstract}
Optoelectronic properties of CVD graphene are characterized over a wide frequency range: THz, IR, visible and near-UV. We used Raman spectroscopy to characterize the synthesized graphene films. All graphene layers were deposited on various substrates, some ones transparent or flexible, such as polyethylene terephthalate (PET), polyethylene naphthalate (PEN), quartz and silicon. Transmission Terahertz time-domain spectroscopy (THz-TDS)
\end{abstract}

method, in the range from $100 \mathrm{GHz}$ to $3 \mathrm{THz}$, is used to analyze the transmittance, sheet conductivity and attenuation of graphene and the complex refractive index of substrates. From IR, near-UV and visible spectroscopy we obtained the transmittance of the substrate and the sample at those frequency ranges, and we deduced the graphene transmittance on each substrate. We found that it is close to $97 \%$ in most cases.
1 Introduction Graphene, a two-dimensional monolayer with a honeycomb lattice structure of carbon atoms, has attracted tremendous interest because of its excellent electrical properties and its potential in nanoelectronic devices.

Terahertz $(\mathrm{THz})$ radiation is very attractive with great potential for applications in biomedical imaging, biology, security, military industry, material science and astronomy. The basic science involved has been also object of different studies [1,2].

The infrared spectroscopy (IR) analysis is important for its role in optoelectronic implementations. Graphene characterization on the $\mathrm{THz}$ and IR bands is a crucial step for its development on optoelectronic devices. Moreover, the comparison between the THz, IR, near-UV and visible band spectroscopy results, for example the transmittance, will give as a complementary information, that has to behave in good agreement.

Transmittance of graphene at the visible band is interesting to measure its transparency. Transparent and flexible substrates like PET and PEN will be useful in flexible electronic devices as sensors or wearables. Transparent electrodes are interesting for different applications, in particular electromagnetic shielding and photovoltaic solar cells.

2 Graphene samples. Growth, transfer and Raman characterization We analyzed an overall of 11 samples of graphene transferred on to various substrates, such as polyethylene terephthalate (PET), polyethylene naphthalate (PEN), quartz and silicon.

Table 1 Summary of samples $S_{i}$, graphene on the substrate, analyzed in the current study. Sample identification are used for later reference.

\begin{tabular}{cccc}
\hline Substrate & Sample & Graphene form & $\begin{array}{c}\text { Number of } \\
\text { samples }\end{array}$ \\
\hline PET & $\mathrm{S}_{1}$ & CVD & 4 \\
PEN & $\mathrm{S}_{2}$ & CVD & 1 \\
Silicon & $\mathrm{S}_{3}$ & CVD & 4 \\
Quartz & $\mathrm{S}_{4}$ & CVD & 1 \\
Quartz & $\mathrm{S}_{5}$ & CVD-IQS & 1 \\
\hline
\end{tabular}

Graphene are obtained by chemical vapor deposition (CVD) and transferred onto different substrates, table 1. Samples $S_{1}$ and $S_{3}$ are obtained at Sejong University as described in [3], $S_{2}$ and $S_{4}$ are from Graphenea (by standard CVD) and sample $S_{5}$ (CVD-IQS graphene) is obtained by us at Institut Quimic de Sarrià, U. Ramón Llull (IQS) using an original CVD based method described briefly on the next section.

2.1 CVD graphene obtained at IQS Graphene is grown in a tubular quartz reactor using copper foil $(25 \mu \mathrm{m}$ thick from Alfa Aesar) as a catalyst. The process can be break down in 4 consecutive steps: (i) rapid heating from 
room temperature to $950^{\circ} \mathrm{C}$, (ii) 15 minutes annealing at $950^{\circ} \mathrm{C}$ under a hydrogen flow of $30 \mathrm{sccm}$, (iii) 25 minutes graphene growth at $950^{\circ} \mathrm{C}$ under $90 \mathrm{sccm}$ of a $30: 70 \mathrm{mix}-$ ture of methane and hydrogen, (iv) rapid quenching to room temperature.

Later on, graphene is transferred from its copper growth substrate on to a quartz plate using the ferric chloride method. First, graphene/copper substrate is spincoated with PMMA, and then copper is dissolved using a $3 \mathrm{M}$ ferric chloride solution. PMMA/graphene is then transferred to a deionized water bath to eliminate ferric chloride residues before placing onto the quartz plate. Finally, PMMA is dissolved away using acetone. Details will be published elsewhere [4].

\subsection{Raman characterization Raman spectroscopy}
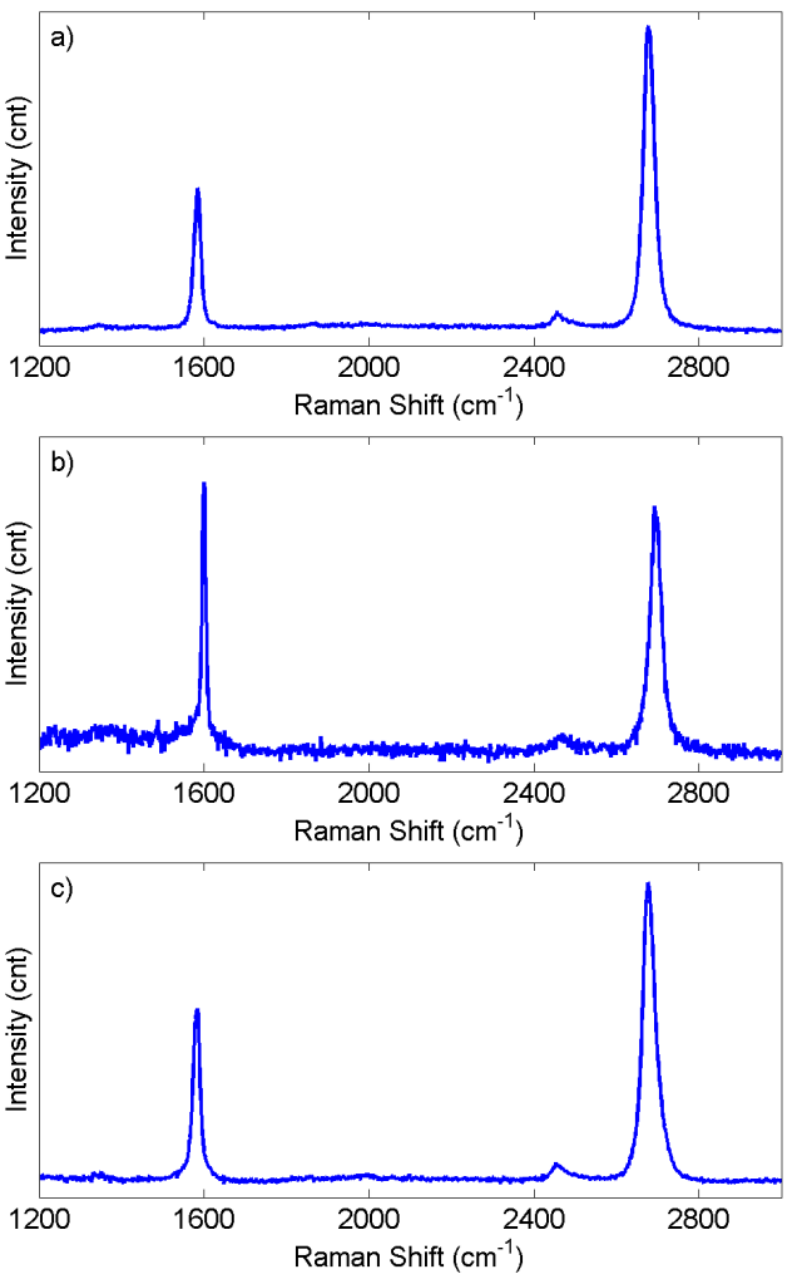

Figure 1 (Color online) Raman spectra of (a) CVD graphene on silicon, $\mathrm{S}_{3}$, (b) CVD graphene on quartz, $\mathrm{S}_{4}$, and (c) CVD-IQS graphene on quartz, $\mathrm{S}_{5}$.

is used with a $532.058 \mathrm{~nm}$ excitation laser with a power of $0.5 \mathrm{~mW}$, acquisition time 20 seconds, on $3-5$ different points, in order to perform a quick sample identification.
The Raman spectra of graphene are well known. Three main typical lines are observed: D line, around $1345 \mathrm{~cm}^{-1}$; $\mathrm{G}$ line, around $1585 \mathrm{~cm}^{-1}$; and 2D line, around $2685 \mathrm{~cm}^{-1}$. The 2D-G intensity ratio lines are usually used to provide a preliminary estimation of the number of layers of graphene. A ratio between 1.5 and 3.5 is usually interpreted as a monolayer-graphene [5,6].

Raman spectra of CVD graphene deposited on PET or PEN, samples $S_{1}$ and $S_{2}$, are not shown because the Raman lines of these polymers overlap with the three main lines of graphene. Therefore, Raman spectra of graphene on PET or PEN could not be provided.

CVD graphene transferred on to silicon, $\mathrm{S}_{3}$, and CVDIQS graphene on quartz, $S_{5}$, present a $2 \mathrm{D}-\mathrm{G}$ ratio lines above 1.5 , so we can determine these samples are monolayer graphene on their substrates.

CVD graphene on quartz, $\mathrm{S}_{4}$, presents a $2 \mathrm{D}-\mathrm{G}$ ratio line below to 1 , therefore this sample is probably a bilayer or trilayer graphene on the substrate.

\section{Experimental}

3.1 THz-Time Domain Spectroscopy Terahertz time-domain spectroscopy (THz-TDS) is a non-destructive testing method based on a coherent detection scheme, implementing a phase-sensitive technique that enables broadband measurements of complex material properties at $\mathrm{THz}$ frequencies, up to $3 \mathrm{THz}$. The $\mathrm{THz}$ measurements are performed in a THz-TDS spectrometer in transmission setup, based on the commercial TERA K8, from Menlo Systems.

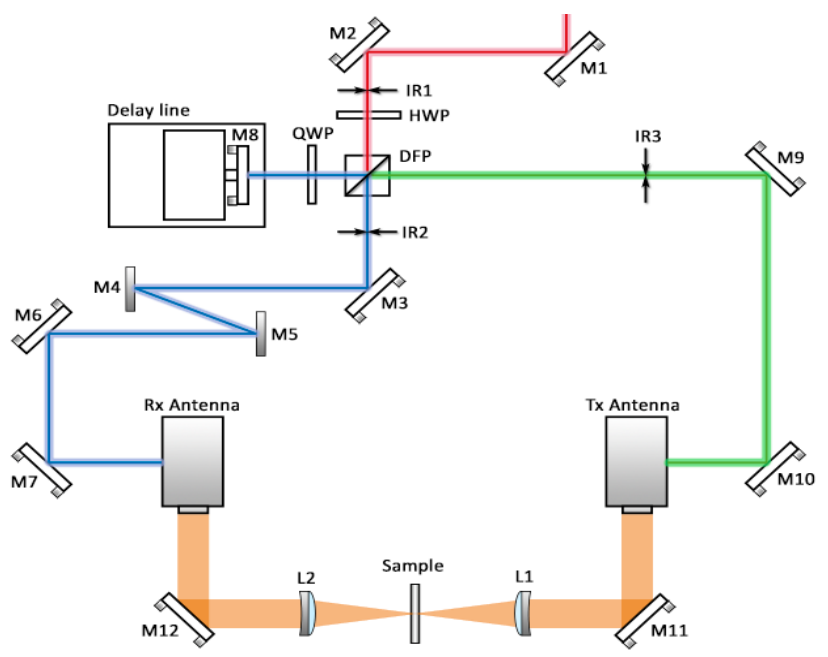

Figure 2 THz-TDS transmission setup configuration.

The broadband $\mathrm{THz}$ radiation is generated using a 780 $\mathrm{nm}$ wavelength fs laser and it is detected with a photoconductive antenna. The output of the laser is split into a pump (generating) and a probe (detecting) beams by a polarizing beam splitter, travelling through two different optical paths to the emitter and the detector antenna, respectively. One path has a variable length, in order to control the pulses delay arriving to the corresponding antenna. 
The transmitted radiation through the sample is focused on a $\mathrm{THz}$ detector antenna which is gated by the probe (detection) laser beam. Finally the recorded time trace is transferred into frequency domain by Fourier transform (FFT) for spectroscopic analysis. The conductance and attenuation of the sample can be deduced.

\subsection{THz-TDS experimental results}

We used $\mathrm{THz}$ time-domain spectroscopy to analyze the photoconductivity of both CVD graphene and CVD-IQS graphene at high frequencies, from $100 \mathrm{GHz}$ to $3 \mathrm{THz}$, deposited on different substrates: PET, PEN, quartz and silicon.

THz-TDS provides a time domain electric field, see figure 3, from which we obtain its $\mathrm{THz}$ spectrum by applying the FFT on the time signal. Therefore, we obtain information of both the amplitude and the phase of the $\mathrm{THz}$ wave in the frequency domain.

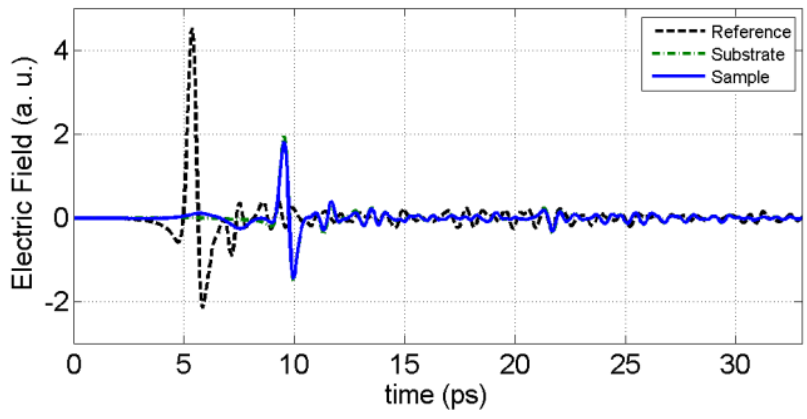

Figure 3 (Color online) Measured temporal electric field pulse of the transmitted $\mathrm{THz}$ wave through air (reference), the substrate and the sample composed by a substrate and graphene deposited on its surface, corresponding to sample $\mathrm{S}_{3}$.

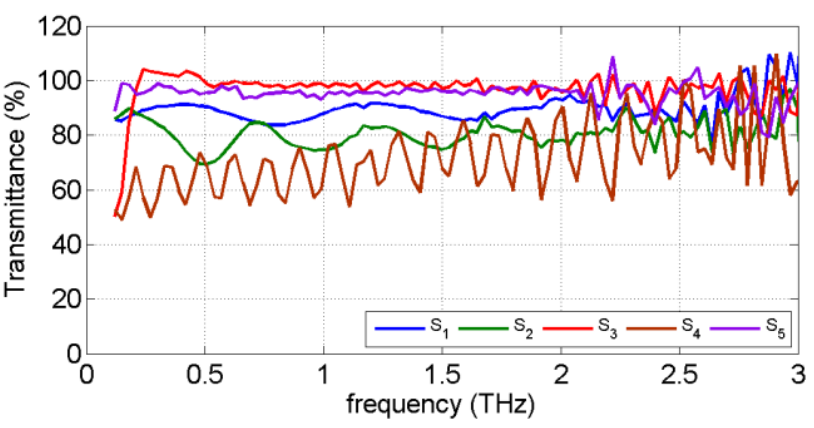

Figure 4 (Color online) Transmittance of graphene. On the left, from top to bottom: $\left(S_{5}\right)$ CVD-IQS graphene on quartz, $\left(S_{2}\right)$ CVD graphene on PEN, $\left(\mathrm{S}_{1}\right)$ CVD graphene on PET $\left(\mathrm{S}_{3}\right)$ CVD graphene on silicon and $\left(\mathrm{S}_{4}\right)$ CVD graphene on quartz.

Transmittance represents the intensity fraction of the $\mathrm{THz}$ wave transmitted through a material and is defined as the ratio between the frequency domain intensity wave transmitted through the sample and the air reference wave, as follows:

$$
T(\omega)=\frac{F F T[e(t)]}{F F T\left[e(t)_{\text {reference }}\right]}
$$

Graphene transmittance, figure 4, is obtained from the ratio of the sample and the bare substrate transmittance:

$$
T(\omega)_{\text {graphene }}=\frac{T(\omega)_{\text {graphene+ substrate }}}{T(\omega)_{\text {substrate }}}
$$

The oscillations seen on figure 4 are related to the substrate thickness, due to the internal Fabry-Pérot reflections.

Monolayer graphene $\left(\mathrm{S}_{1}, \mathrm{~S}_{3}, \mathrm{~S}_{5}\right)$ transmittance in the $\mathrm{THz}$ range is greater than $95 \%$ for $\mathrm{S}_{3}, \mathrm{~S}_{5}$ and close to $90 \%$ for $S_{1}$. Multilayer graphene $\left(S_{2}, S_{4}\right)$ show a transmittance in the range from $60 \%$ to $80 \%$.

From the transmittance of bare substrate and the sample, we can determine a large set of optoelectronic properties, such as the complex refractive index, figure 5, the sheet conductivity of graphene, figure 6 , and the attenuation, figure 7, among others [7].

The complex refractive indices of the different substrates are initially characterized by comparing the signal spectrum with the reference spectrum, minimizing the internal Fabry-Pérot reflections produced in thin films [8]. The real part of the obtained refractive indices, shown on Table 2, are coherent either with the typical values found on bibliography and the values given by the manufacturer.

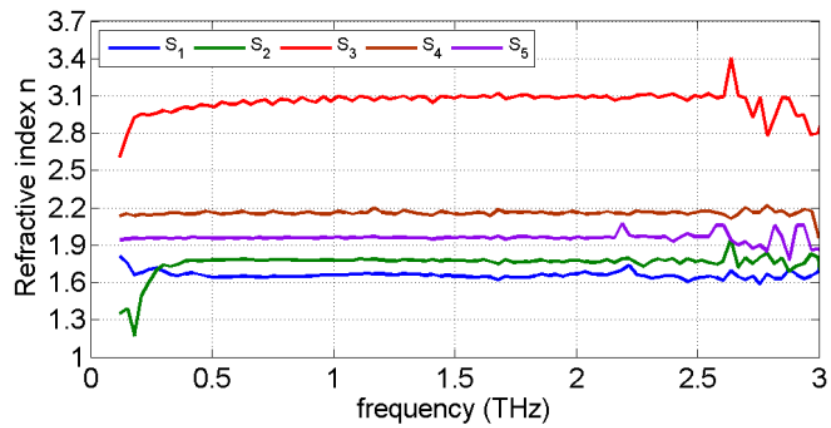

Figure 5 (Color online) Real part of the refractive indices of substrates. On the left, from top to bottom: $\left(\mathrm{S}_{3}\right)$ silicon, $\left(\mathrm{S}_{4}\right)$ quartz, $\left(\mathrm{S}_{5}\right)$ quartz, $\left(\mathrm{S}_{1}\right)$ PET and $\left(\mathrm{S}_{2}\right)$ PEN.

All substrates have to be initially characterized by extracting from the transmittance the complex refraction index in order to be able to evaluate the sheet conductivity of graphene [8].

The conductivity of graphene is usually reported versus its minimum value known as the minimal conductivity of graphene, $\sigma_{\min }$. There are some discrepancies about its value $[9,10]$. In this study, we are considering the value of the minimal conductivity of graphene as:

$$
\sigma_{\min }=\frac{\pi e^{2}}{2 h} \approx 60.85 \mu S_{s q}
$$


We measured the sheet conductivity of graphene deposited on different substrates. To measure the graphene conductivity, we have treated graphene as boundary condition with finite surface conductivity [8].

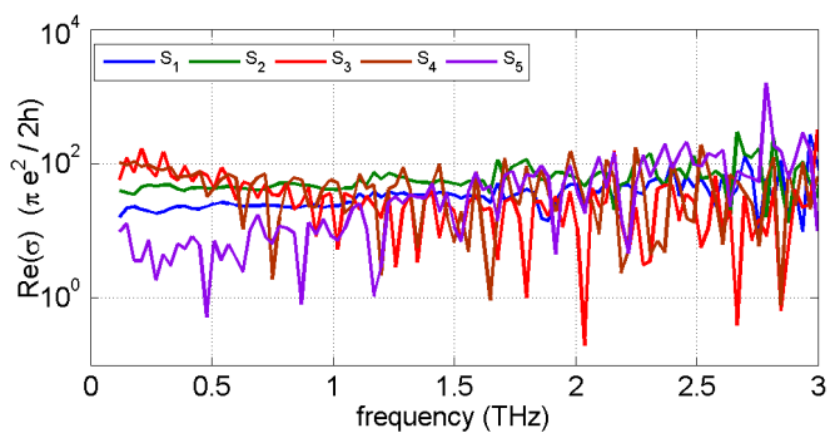

Figure 6 (Color online) Sheet conductivity of graphene. On the left, from top to bottom: $\left(\mathrm{S}_{4}\right)$ CVD graphene on quartz, $\left(\mathrm{S}_{3}\right) \mathrm{CVD}$ graphene on silicon, $\left(\mathrm{S}_{2}\right)$ CVD graphene on PEN, $\left(\mathrm{S}_{1}\right)$ CVD graphene on PET and $\left(\mathrm{S}_{5}\right)$ CVD-IQS graphene on quartz.

Figure 6 shows that CVD graphene present a sheet conductivity around 20 to 90 times the value of minimum conductivity of graphene at frequencies of $\mathrm{THz}$, as expected from previous studies $[11,12]$. However, we found that CVD-IQS graphene shows a sheet conductivity around 2 to 10 times the value of minimum conductivity at relative low frequencies, up to $1.2 \mathrm{THz}$, and 20 to 90 times at higher values, as CVD graphene. This means CVD-IQS graphene is in the same range of conductivity as CVD graphene except for frequencies lower than $1.2 \mathrm{THz}$, where CVD-IQS graphene show a lower sheet conductivity than the other CVD graphene, samples $S_{1}, S_{2}, S_{3}$ and $S_{4}$.

Table 2 Real part of refractive indices of the substrates, sheet conductivity of graphene $\sigma_{\mathrm{S}}(\mu \mathrm{S})$ and normalized sheet conductivity $\sigma_{\mathrm{n}}$ of graphene at $1 \mathrm{THz}$ and $2.5 \mathrm{THz}$, normalized to the minimum value of graphene $\sigma_{\text {min }}$.

\begin{tabular}{ccccccc}
\hline \multicolumn{2}{l}{ SubstrateSample } & $\mathrm{n}$ & $\begin{array}{c}\sigma_{\mathrm{n}} \\
(1 \mathrm{THz})\end{array}$ & $\begin{array}{c}\sigma_{\mathrm{s}} \\
(1 \mathrm{THz})\end{array}$ & $\begin{array}{c}\sigma_{\mathrm{n}} \\
(2.5 \mathrm{THz})\end{array}$ & $\begin{array}{c}\sigma_{\mathrm{s}} \\
(2.5 \mathrm{THz})\end{array}$ \\
\hline PET & $\mathrm{S}_{1}$ & 1.65 & 23.32 & 14.19 & 51.14 & 31.12 \\
PEN & $\mathrm{S}_{2}$ & 1.78 & 41.46 & 25.23 & 91.76 & 55.84 \\
Silicon & $\mathrm{S}_{3}$ & 3.1 & 35.95 & 21.88 & 13.29 & 8.09 \\
Quartz & $\mathrm{S}_{4}$ & 2.15 & 56.87 & 34.61 & 41.8 & 25.44 \\
Quartz & $\mathrm{S}_{5}$ & 1.96 & 8.53 & 5.19 & 93.72 & 57.03 \\
\hline
\end{tabular}

We measured the attenuation of both CVD graphene and CVD-IQS graphene. Attenuation is defined as the intensity loss wave propagation, as follows:

$$
A(\omega)=20 \log _{10}(|T(\omega)|)
$$

Attenuation of graphene is shown on figure 7 . We found that CVD mono-layer graphene, $S_{1}$ and $S_{3}$, present an attenuation below than $1 \mathrm{~dB}$ and CVD-IQS graphene, $\mathrm{S}_{5}$, shows an attenuation below $0.5 \mathrm{~dB}$.
By Raman spectroscopy we found a bilayer or trilayer CVD graphene deposited on quartz substrate, sample $S_{4}$. This is the reason of the increased attenuation with respect to monolayer graphene.

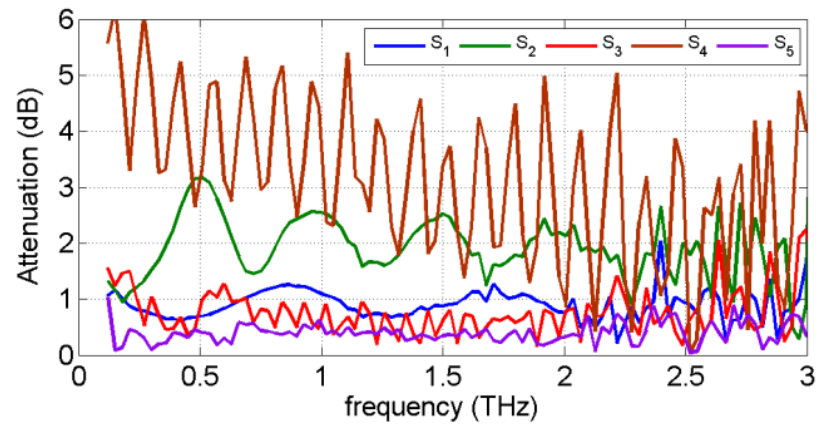

Figure 7 (Color online) Attenuation of graphene. On the left, from top to bottom: $\left(S_{4}\right)$ CVD graphene on quartz, $\left(S_{3}\right)$ CVD graphene on silicon, $\left(S_{2}\right)$ CVD graphene on PEN, $\left(S_{1}\right)$ CVD graphene on PET and $\left(\mathrm{S}_{5}\right)$ CVD-IQS graphene on quartz.

3.3 Infrared spectroscopy We studied graphene transmittance in the infrared band, in the range from 349 $\mathrm{cm}^{-1}$ to $4000 \mathrm{~cm}^{-1}$. Measures have been taken with the FTIR-FTLA 2000 analyzer from ABB at CCiTUB.
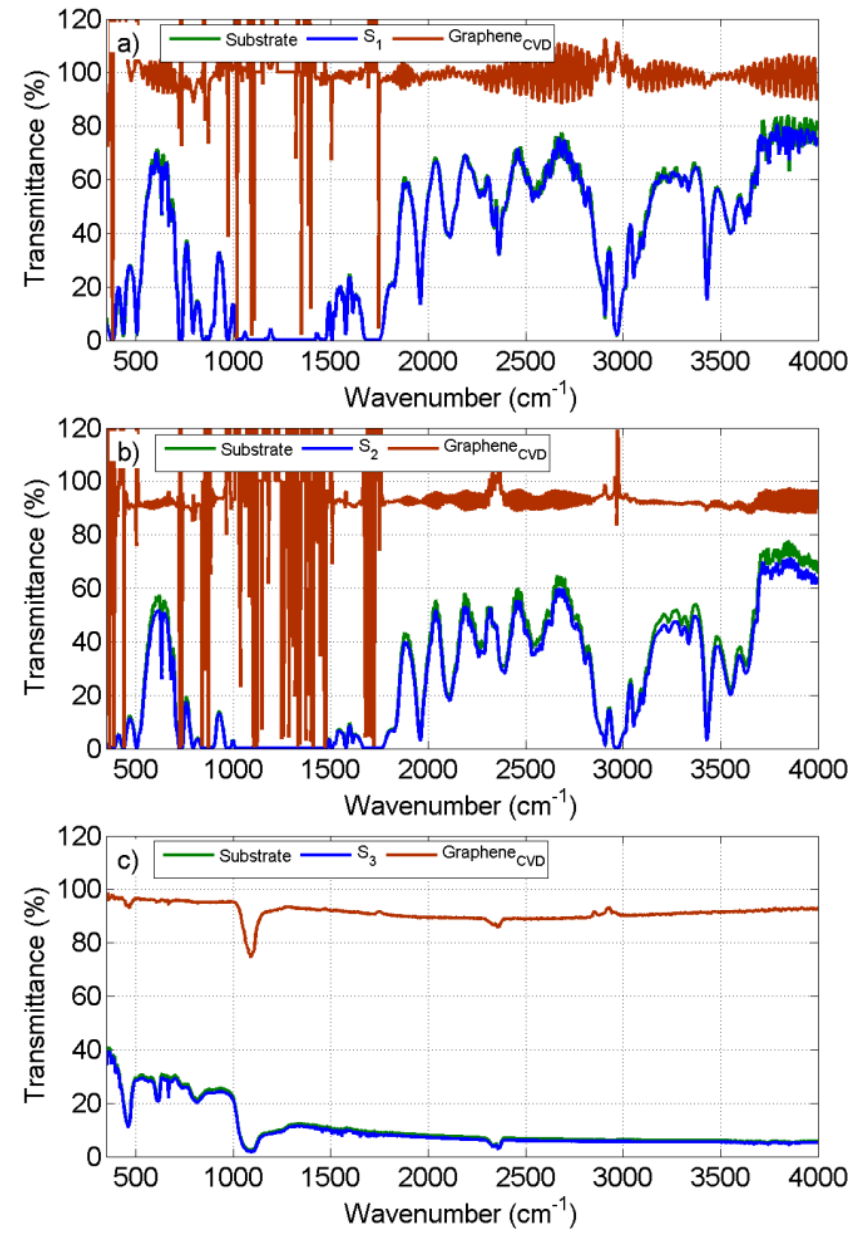



Figure 8 (Color online) IR transmittance, from top to bottom, of graphene, substrate and the sample for different substrates: (a) CVD graphene on PET, $S_{1}$, (b) CVD graphene on PEN, $S_{2}$, (c) CVD graphene on silicon, $S_{3}$, (d) CVD graphene on quartz, $S_{4}$, and (e) CVD-IQS graphene on quartz, $\mathrm{S}_{5}$.

On figure 8 we observe graphene present transmittance values higher than $90 \%$ at the IR band. Transmittance of samples, composed by a substrate and graphene, shows a transmittance very close to the bare substrate transmittance, showing lower values at high wavenumbers.

3.4 UV-Visible spectroscopy We studied both CVD graphene and CVD-IQS graphene for all samples and the substrates in the range from $190 \mathrm{~nm}$ to $1100 \mathrm{~nm}$, see figure 9 , corresponding to near ultraviolet and visible spectra. Measures have been taken with the Analytik Jena Specord 205 system at CCiTUB.

Silicon transmittance is not shown because of its opacity in the frequency range of study.

Considering the fact that graphene transmittance is extracted by comparison (equation 2) using the transmittance of the substrate, we can only determine its transmittance as long as both the transmittance of bare substrate and the sample are not null. Therefore, to represent the graphene transmittance across the range of study, it is necessary that the material does not present a considerably absorption over these frequency range. Particularly, the available material that fulfills better this requirement is quartz.
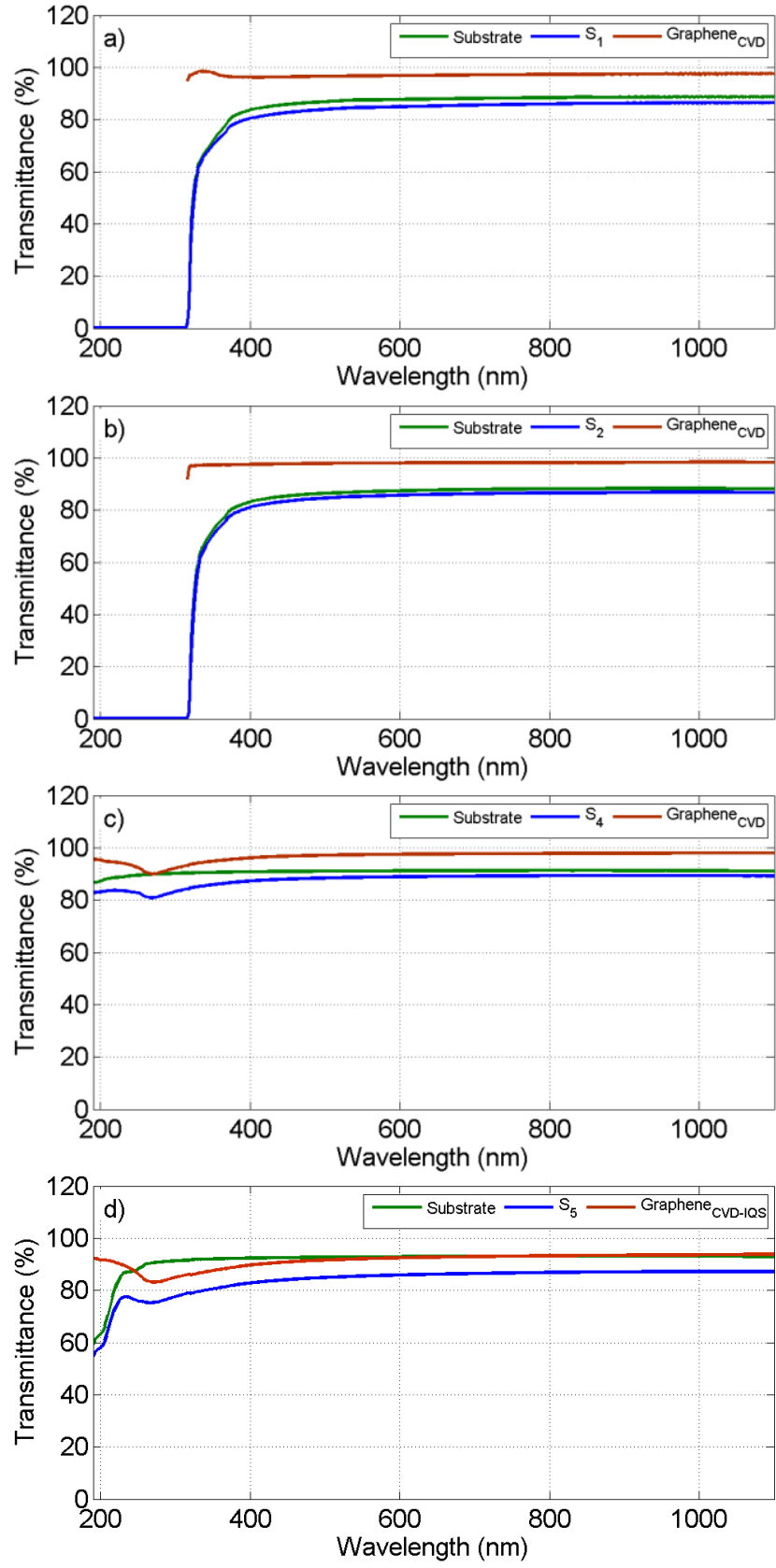

Figure 9 (Color online) UV-Visible transmittance. On the left, from top to bottom, of graphene, substrate and the sample for different substrates: (a) CVD graphene on PET, $S_{1}$, (b) CVD graphene on PEN, $S_{2}$, (c) CVD graphene on quartz, $S_{4}$, and (d) CVD-IQS graphene on quartz, $S_{5}$.

We found an absorption peak at $270 \mathrm{~nm}$, see figure 10 . This peak is produced as a result of the inherent dispersion that takes place in graphene [13]. The peak is visible only for samples S4 and S5, graphene on quartz substrate. The other substrates, PET, S1, or PEN, S3, are only transparent at wavelengths over $314 \mathrm{~nm}$. 


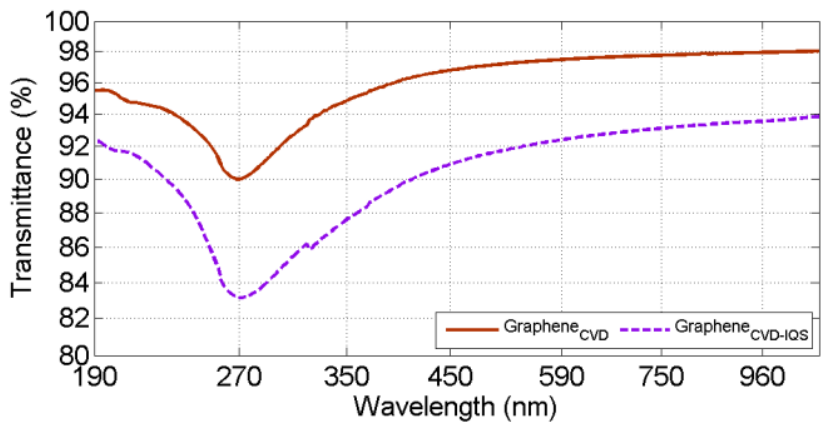

Figure 10 (Color online) We observed an absorption peak centered at a wavelength of $270 \mathrm{~nm}$.

To study the transparency property of graphene for its use in optoelectronic applications, we measure its transmittance at $550 \mathrm{~nm}$, where the spectral response of a human eye is maximum.

Table 3 shows the transmittance (T) of both CVD graphene, CVD-IQS graphene, substrate and the ensemble graphene-substrate. The obtained $\mathrm{T}$ values, as expected, are in good agreement to the reported results on previous studies [14]. In contrast, CVD-IQS graphene shows a reduced value of transmittance compared to other CVD graphene.

Table 3 Transmittance of substrate, graphene and the ensemble graphene-substrate at $550 \mathrm{~nm}$.

\begin{tabular}{ccccc}
\hline Transmittance & Sample & Substrate & $\begin{array}{c}\text { Graphene- } \\
\text { substrate }\end{array}$ & Graphene \\
\hline PET & $\mathrm{S}_{1}$ & $87.44 \%$ & $85 \%$ & $96.7 \%$ \\
PEN & $\mathrm{S}_{2}$ & $87 \%$ & $85.24 \%$ & $97.98 \%$ \\
Quartz & $\mathrm{S}_{4}$ & $91,12 \%$ & $88.7 \%$ & $97.35 \%$ \\
Quartz & $\mathrm{S}_{5}$ & $92.79 \%$ & $85.47 \%$ & $92.11 \%$ \\
\hline
\end{tabular}

4 Discussion and conclusions We have characterized optoelectronic properties of CVD graphene and CVD-IQS graphene over a wide frequency range: THz, IR, visible and near-UV.

We found that a single layer CVD graphene shows a sheet conductivity in the range from 20 to 90 times the value of minimum conductivity of graphene. In contrast, CVD-IQS graphene shows a lower conductivity at frequencies below 1.2 THz. Specifically, CVD-IQS graphene presents a conductivity around 2 to 10 times the value of minimum conductivity at relative low frequencies, up to $1.2 \mathrm{THz}$, and 20 to 90 times at higher values, as other CVD graphene.

The attenuation of graphene in the $\mathrm{THz}$ range is presented. We observed that a single layer CVD graphene presents an attenuation of approximately $1 \mathrm{~dB}$ and the increase of the number of layers of graphene corresponds to an increase in its total attenuation. CVD-IQS graphene shows a slightly lower attenuation than CVD graphene, around $0.5 \mathrm{~dB}$.
We studied the graphene transmittance on the near-UV and visible frequency range and we found that graphene presents an absorption peak at $270 \mathrm{~nm}$.

In the visible range, the analysis of graphene transmittance at $550 \mathrm{~nm}$ reveals that by depositing graphene on a transparent substrate such as PET, PEN or quartz, we can obtain a material that presents a decrement on its transmittance at $550 \mathrm{~nm}$ less than $2.5 \%$, compared to the transmittance of the bare substrate, with values higher than $85 \%$. At the present time, indium tin oxide (ITO) is one of the most used materials in the development of transparent conducting films, with a transmittance around 80-85\% [15] If we compare the transmittance values of ITO and our samples, we found that by depositing graphene on a transparent substrate such as PET, PEN or quartz, we obtain a material that shows a higher transmittance at $550 \mathrm{~nm}$ than ITO, with a similar or even higher conductivity, that can be flexible when deposited on a flexible substrate. Therefore, the use of graphene is reported to be interesting in the development of transparent or flexible optoelectronic devices.

Acknowledgements The authors thank the CCiTUB services for Raman, IR and UV spectroscopy facilities. And M. Z Iqbal and J. Eom at Sejong University for samples. B. Sampere thanks the Spanish Grant (2015 FI_B2 00107).

\section{References}

[1] M. Tonouchi, Nature Photonics 1, 97 (2007).

[2] X. C. Zhang and J. Xu, Introduction to THz Wave Photonics (Springer, 2010).

[3] M. Farooq Khan, M. Zahir Iqbal, M. Waqas Iqbal et al., RSC Adv., 5, 50040 (2015).

[4] B. Sampere, C. Colominas et al., in preparation.

[5] A. C. Ferrari, J. C. Meyer, et al., Phys. Rev. Lett. 97, 187401 (2006)

[6] I. Childres, L. A. Jauregui, W. Park, H. Cao and Y. P. Chen, New developments in photon and materials research (Nova Science Publishers, 2013), chap. 19.

[7] D. Gabriel, Master thesis, ETSETB, UPC (July 2015).

[8] M. Liang, M. Tuo and H. Xin in: $7^{\text {th }}$ European Conference on Antennas and Propagation (EuCAP), Gothenburg, Sweden, 2013, pp. 705-706.

[9] S. Ryu, C. Mudry, A. Furusaki and A. W. W. Ludwig, Phys. Rev. B 75, 205344 (2007).

[10] K. Ziegler, Phys. Rev. B 75, 233407 (2007).

[11] W. Liu, R. Valdés Aguilar, Y. Hao, R. S. Ruoff and N. P. Armitage, J. Appl. Phys. 110 (2012).

[12] N. Rouhim, D. Jain, S. Capdevila, L. Jofre, E. Brown and P. J. Burke in: $11^{\text {th }}$ IEEE International Conference on Nanotechnology (IEEE-NANO), Portland, USA, 2011.

[13] D. Li, B. Muller, S. Gilje, R. B. Kaner and G. G. Wallace, Nat. Nanotechnol. 3, pp. 101-105 (2008).

[14] F. Bonaccorso, Z. Sun and A. C. Ferrari, Nature Photonics 4, $611(2010)$.

[15] D. Kim, Trans. Electr. Electron. Mater. 10 (5), 165 (2009). 